\title{
Dipeptidyl peptidase 4 (DPP4), adipose inflammation, and insulin resistance: is it time to look to the hepatocyte?
}

\author{
Wellington S. Silva Júnior ${ }^{1}$, Maria das Graças C. Souza ${ }^{2}$, Luiz Guilherme Kraemer-Aguiar ${ }^{2,3,4}$ \\ ${ }^{1}$ Endocrinology Discipline, Faculty of Medicine, Center of Natural, Human, Health, and Technology Sciences, Federal University of Maranhão \\ (UFMA), Pinheiro, MA, Brazil; ${ }^{2}$ Laboratory for Clinical and Experimental Research on Vascular Biology (BioVasc), Biomedical Center, State \\ University of Rio de Janeiro (UERJ), Rio de Janeiro, RJ, Brazil; ${ }^{3}$ Postgraduate Program in Clinical and Experimental Physiopathology (FISCLINEX), \\ Faculty of Medical Sciences, UERJ, Rio de Janeiro, RJ, Brazil; ${ }^{4}$ Endocrinology, Department of Internal Medicine, Faculty of Medical Sciences, \\ UERJ, Rio de Janeiro, RJ, Brazil \\ Correspondence to: Luiz Guilherme Kraemer-Aguiar. Department of Internal Medicine, Faculty of Medical Sciences, State University of Rio de \\ Janeiro, Rio de Janeiro, RJ 20550-013, Brazil. Email: lgkraemeraguiar@gmail.com.
}

Submitted Oct 12, 2018. Accepted for publication Oct 17, 2018.

doi: 10.21037/hbsn.2018.10.05

View this article at: http://dx.doi.org/10.21037/hbsn.2018.10.05

Dipeptidyl peptidase 4 (DPP4), also known as adenosine deaminase binding protein or cluster of differentiation 26 (CD26), is a serine exopeptidase able to inactivate peptides composed of proline, hydroxyproline, or alanine as the penultimate residue. It has a strong capacity to act in various peptides and is also widely expressed in many specialized cell types, such as endothelial cells, macrophages, and adipocytes. On its physiological aspects, DPP4 inactivates the glucagon-like peptide-1 (GLP-1), an incretin secreted by the gastrointestinal tract (1). Based on the antidiabetic actions of this incretin, several DPP4 inhibitors (named as gliptins) were launched in the market and are being in use for the treatment of type 2 diabetes (1). It is noteworthy that DPP4 also inactivate some cytokines, chemokines, and neuropeptides involved in inflammation, immunity, and vascular function (2).

A recent article published by Ghorpade et al. (3) puts light on the clinically evident interaction between liver and visceral adipose tissue (VAT) in obesity-induced metabolic diseases. This crosstalk may involve many unknown circulating factors. Inflammation of VAT in obesity is an already well-recognized pathological process, but the source of this inflammation is still a matter of investigation, and maybe some hepatic-derived circulating factor (a hepatokine) would be involved on it. Ghorpade et al. (3) evidenced that obesity in mice stimulates hepatocytes to synthesize and secret DPP4, which acts with plasma factor $\mathrm{Xa}$ to promote inflammation of adipose tissue macrophages and insulin resistance. They demonstrated that soluble DPP4 activates the caveolin-1 pathway in adipose tissue macrophages. In combination with the protease-activated receptor 2 pathway activation by factor $\mathrm{Xa}$, both pathways synergistically stimulate the extracellular signal-regulated protein kinases 1 and 2 and the nuclear factor kappa $\mathrm{B}$, which are distal inflammatory signaling molecules. This finding expands the traditional view of DPP4 as an endothelial product or an adipokine (4) and invites us to look either at the hepatocyte as a source of DPP4, acting as a hepatokine.

Curiously, Ghorpade et al. experimentally showed that silencing expression of DPP4 on hepatocytes suppressed inflammation of VAT and insulin resistance, but this effect did not occur with sitagliptin, an orally administered DPP4 inhibitor (3). We have observed that constitutive DPP4 activity was associated with markers of endothelial activation and microvascular function in humans (5), and that inhibition of this enzyme was associated with attenuation of endothelial dysfunction and atherogenesis (6). However, gliptins did not reduce major cardiovascular outcomes in cardiovascular safety trials (7). All these findings lead us to speculate that genetic silencing and pharmacological inhibition of DPP4 may have different actions on the atherosclerotic process.

Although Ghorpade et al. suggested that silencing DPP4 expression may have metabolic benefits that are not achievable through "currently available oral DPP4 
inhibitors" (3), it is essential to consider that there are differences in the way in which gliptins interact with the DPP4 (1). Sitagliptin, alogliptin, and linagliptin form noncovalent interactions with residues in the catalytic site of DPP4 while vildagliptin and saxagliptin form a reversible covalent enzyme-inhibitor complex in which there are slow rates of inhibitor binding and dissociation, resulting in a slow enzyme balance between the active and inactive forms (1). This balance may further impact on the possible DPP4 inhibitors' ability to mitigate inflammation and insulin resistance promoted by the hepatocyte-secreted DPP4.

The Ghorpade and co-workers' hypothesis arises some doubts to us since they assured that the anti-inflammatory effect of lowering plasma DPP4 activity by sitagliptin is counteracted by its effect of increasing plasma insulin (3). The enhancement of insulin secretion following DPP4 inhibition occurs in a glucose-dependent fashion due to the greater availability of GLP-1. This effect is substantially different from the compensatory hyperinsulinemia induced by insulin resistance, a state that distinctly affects the insulin signal transduction pathways. Insulin resistance compromises the phosphatidylinositol 3-kinase pathway, related to insulin-stimulated glucose uptake, but not the mitogen-activated protein kinase pathway, which plays a role in inflammatory responses and may partially explain the link between hyperinsulinemia and inflammation. Perhaps one or more DPP4 substrates (or even an agent per se) are implicated in this apparent deleterious effect.

If the role of DPP4 in the crosstalk between hepatocytes and adipose tissue observed in mice (3) is confirmed in humans, gene-silencing based therapies specifically focused on hepatocyte DPP4 expression could represent a complementary (or even more prominent) therapeutic option to type 2 diabetes than the currently available gliptins.

Cite this article as: Silva Júnior WS, Souza MD, KraemerAguiar LG. Dipeptidyl peptidase 4 (DPP4), adipose inflammation, and insulin resistance: is it time to look to the hepatocyte? HepatoBiliary Surg Nutr 2018;7(6):499-500. doi: 10.21037/hbsn.2018.10.05

\section{Acknowledgements}

None.

\section{Footnote}

Conflicts of Interest: The authors have no conflicts of interest to declare.

\section{References}

1. Deacon CF. Dipeptidyl peptidase-4 inhibitors in the treatment of type 2 diabetes: a comparative review. Diabetes Obes Metab 2011;13:7-18.

2. Fadini GP, Avogaro A. Cardiovascular effects of DPP-4 inhibition: beyond GLP-1. Vascul Pharmacol 2011;55:10-6.

3. Ghorpade DS, Ozcan L, Zheng Z, et al. Hepatocytesecreted DPP4 in obesity promotes adipose inflammation and insulin resistance. Nature 2018;555:673-7.

4. Lamers D, Famulla S, Wronkowitz N, et al. Dipeptidyl peptidase 4 is a novel adipokine potentially linking obesity to the metabolic syndrome. Diabetes 2011;60:1917-25.

5. da Silva Júnior WS, Souza MD, Nogueira Neto JF, et al. Constitutive DPP4 activity, inflammation, and microvascular reactivity in subjects with excess body weight and without diabetes. Microvasc Res 2018;120:94-9.

6. Silva Júnior WS, Godoy-Matos AF, Kraemer-Aguiar LG. Dipeptidyl peptidase 4: a new link between diabetes mellitus and atherosclerosis? Biomed Res Int 2015;2015:816164.

7. Mannucci E, Mosenzon O, Avogaro A. Analyses of results from cardiovascular safety trials with DPP-4 inhibitors: cardiovascular outcomes, predefined safety outcomes, and pooled analysis and meta-analysis. Diabetes Care 2016;39 Suppl 2:S196-204. 